\title{
A low-numerical dissipation, patch-based adaptive-mesh-refinement method for large-eddy simulation of compressible flows
}

\author{
C. Pantano, R. Deiterding, D.J. Hill and D.I. Pullin \\ Graduate Aeronautical Laboratories \\ California Institute of Technology \\ 1200 E. California Blvd. 205-45, Pasadena, CA \\ E-mail: cpantano@galcit.caltech.edu
}

\begin{abstract}
This paper describes a hybrid finite-difference method for the large-eddy simulation of compressible flows with low-numerical dissipation and structured adaptive mesh refinement (SAMR). A conservative flux-based approach is described with an explicit centered scheme used in turbulent flow regions while a weighted essentially non-oscillatory (WENO) scheme is employed to capture shocks. Three-dimensional numerical simulations of a Richtmyer-Meshkov instability are presented.
\end{abstract}

\section{Introduction}

Compressible flows of practical interest generally contain physically different key features, e.g. complex shock structures and turbulence. Efficient numerical simulation of these flows typically requires varying degrees of spatial resolution. This need has made structured adaptive mesh refinement (SAMR) techniques very popular for the Euler equations $[1,2]$. Moreover, the regular data decomposition of SAMR enables efficient load balancing on current distributed memory computers. For compressible turbulent flows, it is often expedient to implement a solver appropriate to the locally dominant physics; for example switched or hybrid methods that become upwind-biased around shocks, but which revert to centered stencils in nominally smooth regions. This dual requirement in both resolution and algorithm adaptivity presents a significant challenge for the construction of numerical methods suitable for the large-eddy simulation of strongly compressible turbulence. In this context, flux-based shock-capturing methods are employed to ensure weak convergence (prediction of the correct wave speeds) [3], whereas in turbulent flow-regions methods with low numerical dissipation are preferred. Centered numerical schemes satisfy the latter requirement in a natural manner, but care must be taken to avoid non-linear instabilities since there is no intrinsic numerical stabilization. This problem can be alleviated to some extent by using energy conserving (skew-symmetric) formulations [4].

We describe a hybrid finite-difference solver for large-eddy simulation of compressible flows with low-numerical dissipation with SAMR. This numerical method is used together with the AMROC (Adaptive Mesh Refinement in Object-oriented $\mathrm{C}++$ ) framework [5] that implements the Berger and Colella algorithm. Its primary applications are three-dimensional compressible 
turbulent flows driven by shocks. A flux-based approach is described that remains conservative at fine-coarse mesh interfaces resulting from SAMR, and also in the presence of numerical scheme switching. An explicit, tuned centered discretization (TCD) is used in smooth and turbulent regions of the flow. The TCD scheme transitions smoothly to a weighted essentially nonoscillatory (WENO) method for shock capturing. The entire approach is a further development and significant improvement of the hybrid method of Hill and Pullin [6]. A number of numerical experiments and validations have been conducted elsewhere, ranging from two- to three-dimensional problems that include homogeneous shock free turbulence, turbulent (reacting and non-reacting) jets and the strongly shock driven mixing of a Richtmyer-Meshkov instability (RMI) that is discussed here.

\section{Numerical method}

We solve the compressible LES equations in conservation form. The stretched vortex subgrid model for momentum and scalar transport is used in the present simulations [7, 8]. Mass, momentum, total energy and internal energy variance are discretely conserved using a skewsymmetric formulation for compressible flows [9]. We use an optimized centered second-order finite difference scheme (TCD) [6] given by

$$
\left.\frac{\partial f}{\partial x}\right|_{j}=\frac{1}{\Delta x}\left(\alpha\left(f_{j+2}-f_{j-2}\right)+\beta\left(f_{j+1}-f_{j-1}\right)\right),
$$

where the derivative of a function $f$ is evaluated at discrete uniform intervals with stepsize $\Delta x$. The constants $\alpha, \beta$ are set to the values $\alpha=-0.197, \beta=1 / 2-2 \alpha$, which minimizes LES truncation errors in turbulent regions of Kolmogorov-type. A further aspect is that the SAMR approach is tailored specifically for flux-based discretizations. Numerical fluxes consistent with the centered finite difference stencil are therefore required at the cell edges and, given a stencil of the form Eq. (1), one must derive the corresponding fluxes such that

$$
\left.\frac{\partial f}{\partial x}\right|_{j}=\frac{F_{j+1 / 2}-F_{j-1 / 2}}{\Delta x}
$$

is satisfied. A flux $F_{j+1 / 2}^{d i v}$ which satisfies Eq. (2) for the TCD alone can be obtained readily and reads

$$
F_{j+1 / 2}^{d i v}=\alpha\left(f_{j+2}+f_{j-1}\right)+(\alpha+\beta)\left(f_{j}+f_{j+1}\right) .
$$

Additionally, skew-symmetric terms must be written in flux-conservative form $[10,11]$. Skewsymmetric fluxes $F_{j+1 / 2}^{s k e w}$ are sought satisfying

$$
\left.a \frac{\partial b}{\partial x}\right|_{j}+\left.b \frac{\partial a}{\partial x}\right|_{j} \equiv \frac{F_{j+1 / 2}^{s k e w}-F_{j-1 / 2}^{s k e w}}{\Delta x},
$$

when $a$ and $b$ are replaced by the momentum $\bar{\rho} \tilde{u}_{k}$, the velocity $\tilde{u}_{i}$, the internal energy $\tilde{e}$, the species mass fraction $\tilde{Y}_{i}$, and the pressure $\bar{p}$, depending on the transport equation being considered. It is easily verified that the choice

$$
F_{j+1 / 2}^{s k e w}=\alpha\left(a_{j+2} b_{j}+a_{j-1} b_{j+1}+a_{j} b_{j+2}+a_{j+1} b_{j-1}\right)+\beta\left(a_{j} b_{j+1}+a_{j+1} b_{j}\right),
$$

then satisfies Eq. (4) for the TCD derivative defined in Eq. (1). The total non-dissipative skew-symmetric flux is then given by

$$
F_{j+1 / 2}^{T C D}=\frac{1}{2}\left(F_{j+1 / 2}^{d i v}+F_{j+1 / 2}^{s k e w}\right),
$$




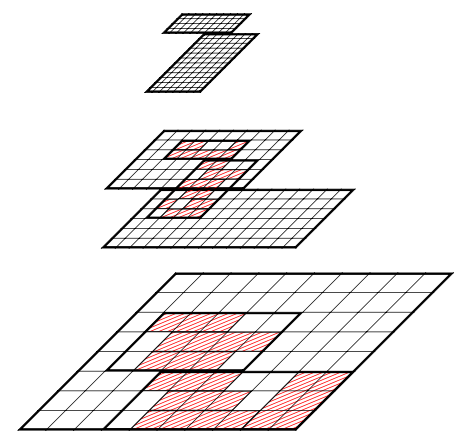

Figure 1. Example of a mesh hierarchy with three levels.

for the momentum and scalar transport equations. A similar expression can be derived for the energy equation. This flux-based approach simplifies the implementation of a consistent and conservative scheme with SAMR considerably [12]. Finally, the fluxes at the physical domain boundaries are computed consistently with the skew-symmetric formulation and the discrete boundary stencil specifically derived for the TCD.

The second part of our hybrid approach involves the WENO scheme. In this case, upwindbiased fluxes, $F_{i+1 / 2}^{W E N O}$, are calculated based on a convex weighting of candidate stencils designed to minimize differentiation across discontinuities. We use the 5-point stencil version of WENO [13] in which the WENO optimal stencil has been replaced by Eq. (1). This modification minimizes dispersion errors produced by the mismatch of modified wavenumber behavior of the standard 5-point WENO scheme when used in hybrid mode together with Eq. (1) [6].

We treat discontinuous flow features and changes in mesh resolution similarly by performing upwind-biased differentiation. Scheme switching at flow discontinuities is achieved through a detection criteria that is problem-dependent; fine-coarse mesh interfaces are flagged directly by AMROC. If flagged regions are denoted by $\mathcal{C}$, the hybrid flux takes the form

$$
F_{i+1 / 2}= \begin{cases}F_{i+1 / 2}^{W E N O}, & \text { in } \mathcal{C} \\ F_{i+1 / 2}^{T C D}, & \text { in } \overline{\mathcal{C}},\end{cases}
$$

where $\overline{\mathcal{C}}$ is the complement of $\mathcal{C}$. Currently, we employ a relative curvature criterion in pressure and density for shock detection.

The SAMR block-structured embedded layout (within AMROC) is shown in figure 1 [5] for a 3 level mesh hierarchy, with a hypothetical flow feature depicted in red. The penalty of this structured approach, as opposed to a purely unstructured technique, is that one looses the kinetic energy conservation property at fine-coarse level interfaces. Finally, the solution is marched in time using a third-order Runge-Kutta method. Characteristic boundary conditions are applied at in- and outflow surfaces.

When SAMR is used in conjunction with LES, purely numerical disturbances may result from the interaction between the flow physics and adaptive refinement, especially if the dynamic mesh adaptation is insufficiently sensitive to some flow features. Spurious waves may arise if vortical unsteady structures, which may be marginally resolved in LES, travel through fine to coarse mesh boundaries. In the traditional SAMR approach, important flow features are always refined appropriately $[1,2]$. Transient features are kept within the refinement by frequent mesh adaptation. In the case of problems involving shocks, one cannot resolve the flow discontinuities and it is therefore necessary to choose an upper bound on the error of the numerically captured discontinuity depending on computational resources and precision needs. Similarly, in LES, 


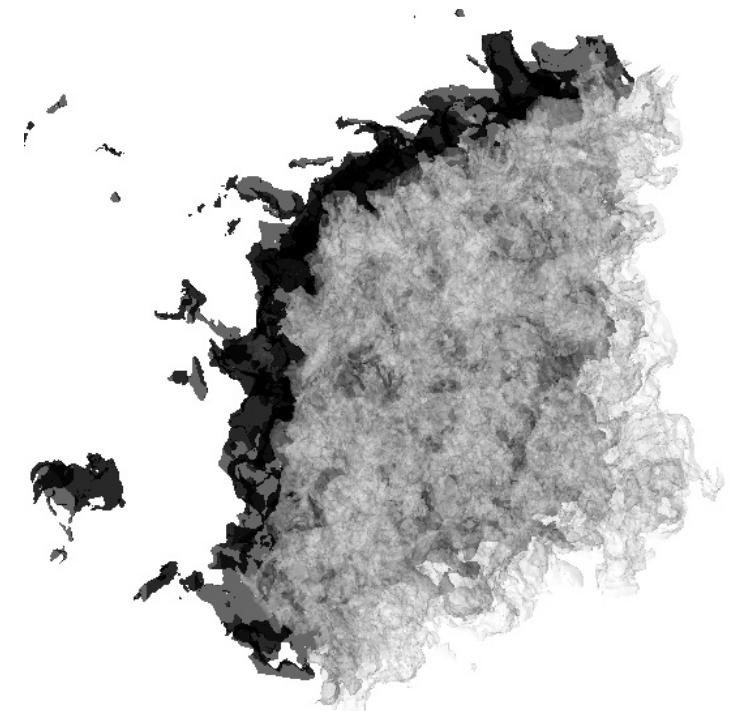

(a) RMI Interface

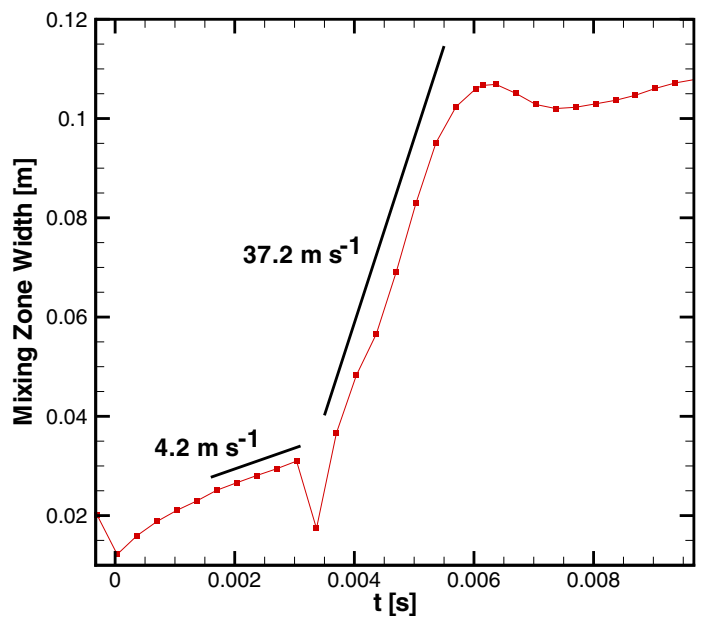

(b) RMI Mixing zone as a function of time (red line) and experimental growth rate (experiment)

Figure 2. Growth rate of RMI.

one would predetermine a certain cutoff scale based on the fraction of turbulence intensity that needs to be captured in the simulation and model the subgrid contributions. In principle, this cutoff scale should be larger than the smallest grid resolution available and independent of the mesh resolution at all levels. This guarantees that the computed LES solution converges to the solution of the LES equations with increasing resolution.

\section{Comparison with experiments of Richtmyer-Meshkov Instability}

We have simulated a variety of shock driven turbulent flows, including the planar re-shock experiment of [14] in which a Mach 1.5 shock interacts with an Air-SF 6 interface and generates turbulent mixing through the Richtmyer-Meshkov instability. The unshocked air has a density of $0.27885 \mathrm{~kg} / \mathrm{m}^{3}$ and pressure of $23 \mathrm{kPa}$. Temperature is uniform in the unshocked side. These flows exercise both the LES and the shock-capturing features of the solver with dynamically adaptive meshes. Visualizations of the Richtmyer-Meshkov instability are shown in figure 2(a). Figure 2(b) shows the thickness of the mixing zone as a function of time and the respective experimental measurement of [14] $\left(\right.$ case $\left.\mathrm{IV}^{e}\right)$. Further statistical results of this flow can be found in [15].

\section{Conclusions}

We have described an extension of the Berger-Colella SAMR method for compressible flows to large-eddy simulation with a hybrid scheme. The formulation uses low-numerical dissipation, centered schemes in skew-symmetric form within patches of uniform resolution in turbulent regions, and upwind-biased differentiation at and around shocks and at fine-coarse mesh interfaces. This operator is based on a modified version of the WENO method whose reference stencil matches the centered scheme. This property is crucial in order to minimize spurious reflections when the scheme transitions from centered to upwind form.

Several verification and test simulations have been analyzed and the order of accuracy, minimal LES-SAMR refinement conditions, and energy generation and loss at fine-coarse mesh interfaces of the overall method have been discussed elsewhere [16]. The presented LES-SAMR 
scheme allowed us to compare simulation results obtained on conventional distributed memory systems of moderate size directly to three-dimensional shock-induced mixing flow produced by the Richtmyer-Meshkov instability.

\section{Acknowledgments}

This work was supported by the Advanced Simulation and Computing (ASC) Program under subcontract no. B341492 of DOE contract W-7405-ENG-48. The authors would like to acknowledge the many helpful conversations with P.E. Dimotakis and D.I. Meiron.

\section{References}

[1] M.J. Berger and J. Oliger. Adaptive mesh refinement for hyperbolic partial-differential equations. J. Comp. Phys., 53(3):484-512, 1984.

[2] M.J. Berger and P. Colella. Local adaptive mesh refinement for shock hydrodynamics. J. Comp. Phys., 82(1):64-84, 1989.

[3] P. Lax and B. Wendroff. Systems of conservation laws. Comm. Pure and Appl. Math., 13(2):217-237, 1960.

[4] T.A. Zang. On the rotation and skew-symmetric forms for incompressible flow simulation. Appl. Numer. Math., 7(1):27-40, 1991.

[5] R. Deiterding. Parallel adaptive simulation of multi-dimensional detonation structures. PhD thesis, Brandenburgische Technische Universität Cottbus., 2003.

[6] D.J. Hill and D.I. Pullin. Hybrid tuned center-difference-WENO method for large eddy simulations in the presence of strong shocks. J. Comp. Phys., 194(2):435-450, 2004.

[7] A. Misra and D.I. Pullin. A vortex-based subgrid model for large-eddy simulation. Phys. Fluids, 9(8):2443-2454, 1997.

[8] D.I. Pullin. Vortex-based model for subgrid flux of a passive scalar. Phys. Fluids, 12(9):2311-2319, 2000.

[9] A.E. Honein and P. Moin. Higher entropy conservation and numerical stability of compressible turbulence simulations. J. Comp. Phys., 201(2):531-545, 2004.

[10] Y. Morinishi, T.S. Lund, O.V. Vasilyev, and P. Moin. Fully conservative higher order finite difference schemes for incompressible flow. J. Comp. Phys., 143(1):90-124, 1998.

[11] F. Ducros, F. Laporte, T. Souleres, V. Guinot, P. Moinat, and B. Caruelle. High-order fluxes for conservative skew-symmetric-like schemes in structured meshes: Application to compressible flows. J. Comp. Phys., 161(1):114-139, 2000.

[12] A. Benkenida, J. Bohbot, and J.C. Jouhaud. Patched grid and adaptive mesh refinement strategies for the calculation of the transport of vortices. Int. J. Numer. Meth. Fluids, 40:855-873, 2002.

[13] G.-S. Jiang and C.-W. Shu. Efficient implementation of weighted ENO schemes. J. Comp. Phys., 126(1):202-228, 1996.

[14] M. Vetter and B. Sturtevant. Experiments on the Richtmyer-Meshkov instability on a air/SF 6 interface. Shock Waves, 4(5):247-252, 1995.

[15] D. J. Hill, C. Pantano, and D. I. Pullin. Large-eddy simulation and multiscale modeling of a richtmyer-meshkov instability with reshock. J. Fluid Mech., 557:29-61, 2006.

[16] C. Pantano, R. Deiterding, D. J. Hill, and D. I. Pullin. A low-numerical dissipation patchbased adaptive mesh refinement method for large-eddy simulation of compressible flows. $J$. Comp. Phys., in press, 2006. 